\title{
The non-abelian normal CM-fields of degree 36 with class number one
}

\author{
by \\ Ku-Young Chang (Taejon) and Soun-Hi Kwon (Seoul)
}

1. Introduction. A. M. Odlyzko proved that there are only finitely many normal CM-fields with class number one $([\mathrm{O}])$. J. Hoffstein proved that their degrees are less than or equal to $436([\mathrm{H}])$. Yamamura has determined all the imaginary abelian number fields with class number one $([\mathrm{Y}])$. According to $[\mathrm{LLO}]$ and $[\mathrm{P}]$ there are seven non-abelian normal CM-fields of degree 24 with class number one. We determine all normal CM-fields of degree $4 p^{2}$ with class number one, where $p$ is an odd prime number.

Theorem 1. (1) If $K$ is a non-abelian normal CM-field of degree $4 p^{2}$ with relative class number one, then $p=3$ and the Galois group of $K$ is isomorphic to $D_{12} \times C_{3}$, the direct product of the dihedral group of order 12 and the cyclic group of order 3 .

(2) There are three non-abelian normal CM-fields $K$ of degree 36 with relative class number one, namely the composita $K=E M k$ given in Table 1 below, where $E=\mathbb{Q}\left(\alpha_{E}\right)$ with $P_{E}\left(\alpha_{E}\right)=0$ is a non-normal totally real cubic field of discriminant $d_{E}, M$ is an imaginary biquadratic bicyclic field $M=$ $\mathbb{Q}\left(\sqrt{-m_{0}}, \sqrt{-m_{1}}\right)$, and $k$ is a cyclic cubic field of conductor $f_{k}$. Moreover, these three fields have class number one.

Table 1

\begin{tabular}{ccccc}
\hline$d_{E}$ & $P_{E}(X)$ & $\left(m_{0}, m_{1}\right)$ & $f_{k}$ & $h_{K^{+}}$ \\
\hline 756 & $X^{3}-6 X-2$ & $(3,7)$ & 9 & 1 \\
1620 & $X^{3}-12 X-14$ & $(3,15)$ & 9 & 1 \\
4312 & $X^{3}+X^{2}-16 X-8$ & $(2,11)$ & 7 & 1 \\
\hline
\end{tabular}

The class numbers $h_{K^{+}}$of the maximal totally real subfields $K^{+}$are obtained by using Kash $([\mathrm{K}])$. Throughout this paper the following notations will be used. For a number field $K$ we let $h_{K}, d_{K}, O_{K}, \omega_{K}$, and $\zeta_{K}$ denote

2000 Mathematics Subject Classification: Primary 11R29; Secondary 11R20. 
the class number, the absolute value of the discriminant, the ring of algebraic integers, the number of roots of unity in $K$, and the Dedekind zeta function of $K$, respectively. If $K$ is a CM-field, we let $h_{K}^{-}, K^{+}$, and $Q_{K} \in\{1,2\}$ denote the relative class number, the maximal totally real subfield, and the Hasse unit index of $K$, respectively (see [W]). We let $\mathfrak{F}_{K_{1} / K_{2}}$ denote the conductor of an abelian extension $K_{1} / K_{2}$. For a primitive Hecke character $\chi$ we let $\mathfrak{F}_{\chi}$ denote the finite part of its conductor.

\section{Normal CM-fields of degree $4 p^{2}$}

Proposition 1. Let $K$ be a non-abelian normal CM-field of degree $4 p^{2}$. Then either $K$ is a dihedral $C M$-field of degree $4 p^{2}$, or $K$ is a dicyclic $C M$ field of degree $4 p^{2}$, or $K=N_{1} N_{2}$ is a compositum of two normal CM-fields $N_{1}$ and $N_{2}$ of degree $4 p$ such that $M=N_{1} \cap N_{2}$ is an imaginary abelian quartic field (notice that each $N_{i}$ is either abelian, or dihedral, or dicyclic).

Proof. Let $S_{p}$ be a $p$-Sylow subgroup of $G$ the Galois group of $K$ over $\mathbb{Q}$, $M$ the fixed subfield of $S_{p}$, and let $c \in G$ denote the complex conjugation. Since $c$ is in the center of $G$ (see [LOO, Lemma 2]), the fixed subfield $M^{+}$of the subgroup $\left\langle c, S_{p}\right\rangle$ is a quadratic field contained in $M$. Hence, if $M$ were not normal then its normal closure $\widehat{M}$ would be a dihedral octic subfield of the normal field $K$ of degree $4 p^{2}$. A contradiction. Therefore, $M$ is a normal quartic field, hence an imaginary abelian quartic field, and $S_{p}$ is a normal subgroup of $G$. Since $S_{p}$ is of order $p^{2}$, it is abelian. If $S_{p}$ is cyclic, then $K / M^{+}$is cyclic of degree $2 p^{2}$ and $K / \mathbb{Q}$ is either dihedral or dicyclic.

Now, assume that $S_{p}=C_{p} \times C_{p}$ is not cyclic. Let $S_{2}$ be a 2-Sylow subgroup of $G$. Since $S_{2}$ acts on the set of the $p+1$ subgroups $C$ of order $p$ of $S_{p}$ by $s_{2} \cdot C=s_{2} C s_{2}^{-1}$ for $s_{2} \in S_{2}$, the number $n$ of such subgroups of order $p$ which are invariant under the action of $S_{2}$ satisfies $p+1 \equiv n(\bmod 2)$ and $n \neq 0$, hence is $\geq 2$. Therefore, there exist at least two distinct normal subgroups $G_{1}$ and $G_{2}$ of order $p$ in $G$. By denoting $N_{i}$ the fixed subfield of $G_{i}$, we obtain the desired result.

Proposition 2. (1) (Theorem 5 of [LOO]) Let $k \subseteq K$ be two CM-fields. If $[K: k]$ is odd, then $Q_{K}=Q_{k}$ and $h_{k}^{-}$divides $h_{K}^{-}$.

(2) (Theorem 7 of [LOO]) There is no dicyclic CM-field of degree $4 p$ with relative class number one.

(3) (Proposition 5.2 of [Lef]) If $N$ is a CM-field of Galois group $D_{2^{m} p^{2}}$, the dihedral group of order $2^{m} p^{2}$, with $m \geq 2$, then $h_{N}^{-}>1$.

Corollary 1. If $K$ is a normal CM-field of degree $4 p^{2}$ with relative class number one, then $\operatorname{Gal}(K / \mathbb{Q})$ is isomorphic to $C_{p} \times D_{4 p}$.

Proof. According to Proposition 2 if $K$ is either a dihedral CM-field of degree $4 p^{2}$ or a dicyclic CM-field of degree $4 p^{2}$, then $h_{K}^{-}>1$. If $K=N_{1} N_{2}$ 
is a compositum of two normal CM-fields $N_{1}$ and $N_{2}$ of degree $4 p$ such that $N_{1} \cap N_{2}$ is an abelian quartic field and if any one of $N_{i}$ 's is dicyclic, then $h_{K}^{-}>1$. If an imaginary abelian number field has relative class number one, then its degree over $\mathbb{Q}$ is not greater than 24 ; therefore $K$ cannot be an abelian number field $([\mathrm{CK}])$. Using $[\mathrm{LOO}]$ and [Lef] we verify that there is no pair of dihedral CM-fields $N_{1}$ and $N_{2}$ of degree $4 p$ such that $N_{1} \cap N_{2}$ is a quartic number field and $h_{N_{1}}^{-}=h_{N_{2}}^{-}=1$. In conclusion, $K$ is a compositum of a dihedral CM-field $N_{1}$ of degree $4 p$ and an abelian number field $N_{2}$ of degree $4 p$ such that $N_{1} \cap N_{2}$ is an imaginary bicyclic biquadratic number field.

3. Normal CM-fields with Galois group $C_{p} \times D_{4 p}$. Let $K$ be a normal CM-field of degree $4 p^{2}$ and Galois group $C_{p} \times D_{4 p}$. Then $K=N_{1} N_{2}$ is a compositum of a dihedral CM-field $N_{1}$ of degree $4 p$ and of an imaginary abelian field $N_{2}$ of degree $4 p$ which are both cyclic extensions of an imaginary biquadratic field $M=\mathbb{Q}\left(\sqrt{-m_{0}}, \sqrt{-m_{1}}\right)$. If $h_{K}^{-}=1$ then $h_{N_{1}}^{-}=h_{N_{2}}^{-}=1$, by Proposition 2(1). Hence, $p=3$ by [Lef, Th. 4.1] and [CK, Table I]. Thus Theorem 1(1) is proved.

Let us now proceed with the proof of Theorem 1(2). According to [Lef, Th. 4.1] or [LOO, Table 1] (in which the 15 th entry should be [ $\left[8505, X^{3}-\right.$ $27 X-51)(7,15) 2]$ ) and according to [CK, Table I], $K$ must be one of the 13 composita $K=N_{1} N_{2}$ of degree 36 given in Table 2 below, where $N_{1}=E M$ is a dihedral CM-field of degree $12, N_{2}=k M$ an imaginary abelian field of degree $12, E=\mathbb{Q}\left(\alpha_{E}\right)$ with $P_{E}\left(\alpha_{E}\right)=0$ is a totally real cubic field, $k$ a real cyclic cubic field, and $M=\mathbb{Q}\left(\sqrt{-m_{0}}, \sqrt{-m_{1}}\right)$ an imaginary biquadratic field. In Table 2 we denote by $L$ the real quadratic subfield of $M$ so that $\operatorname{Gal}(K / L)=C_{2} \times C_{3} \times C_{3}$ and use the following Dirichlet characters to describe the cyclic cubic fields $k$ :

$$
\chi_{7}(3)=e^{2 i \pi / 6}, \quad \psi_{9}(2)=e^{2 i \pi / 3}, \quad \chi_{13}(2)=e^{2 i \pi / 12} .
$$

Now, we use the following proposition to prove that $3^{2}$ divides $h_{K}^{-}$for the seven CM-fields numbered 1, 3, 5, 6, 7, 9 and 10 in Table 2:

Proposition 3 (see Proposition 8 of [LOO]). Let $N / M$ be a cyclic extension of degree $p$ of $C M$-fields. Assume that $N^{+} / M^{+}$is a cyclic extension of degree $p$. Let $t$ be the number of prime ideals of $M^{+}$which split in $M / M^{+}$ and are ramified in $N^{+} / M^{+}$. Then $p^{t-1} h_{M}^{-}$divides $h_{N}^{-}$.

For example let us consider the field $N$ numbered 1 . Then $L=\mathbb{Q}(\sqrt{3})$, 7 is inert in $L$, the ideal $7 O_{L}$ splits completely in $N_{1}$, and $7 O_{L}$ is ramified in $N_{2}^{+}$. Namely, there are at least 3 prime ideals which split in $N_{1} / N_{1}^{+}$and are ramified in $K^{+} / N_{1}^{+}$. Hence $3^{2} \mid h_{N}^{-}$. In the last column of Table 2 we list the appropriate prime ideals. The prime ideal $\mathfrak{p}_{7}$ is the prime ideal lying above 7 in $L$. 
Table 2

\begin{tabular}{|c|c|c|c|c|c|}
\hline No. & $d_{E}$ & $P_{E}(X)$ & $\left(m_{0}, m_{1}\right)$ & $k$ & Prime ideals \\
\hline 1 & \multirow{2}{*}{2700} & \multirow{2}{*}{$X^{3}-15 X-20$} & \multirow{2}{*}{$(1,3)$} & $\chi_{7}^{2}$ & $7 O_{L}$ \\
\hline 2 & & & & $\psi_{9}$ & \\
\hline 3 & \multirow{4}{*}{756} & \multirow{4}{*}{$X^{3}-6 X-2$} & \multirow{4}{*}{$(3,7)$} & $\chi_{7}^{2}$ & $\mathfrak{p}_{7}$ \\
\hline 4 & & & & $\psi_{9}$ & \\
\hline 5 & & & & $\chi_{13}^{4}$ & $13 O_{L}$ \\
\hline 6 & & & & $\chi_{7}^{4} \psi_{9}$ & $\mathfrak{p}_{7}$ \\
\hline 7 & \multirow{2}{*}{1620} & \multirow{2}{*}{$X^{3}-12 X-14$} & \multirow{2}{*}{$(3,15)$} & $\chi_{7}^{2}$ & $7 O_{L}$ \\
\hline 8 & & & & $\psi_{9}$ & \\
\hline 9 & \multirow{2}{*}{1944} & \multirow{2}{*}{$X^{3}-9 X-6$} & \multirow{2}{*}{$(2,3)$} & $\chi_{7}^{2}$ & $7 O_{L}$ \\
\hline 10 & & & & $\chi_{13}^{4}$ & $13 O_{L}$ \\
\hline 11 & 4312 & $X^{3}+X^{2}-16 X-8$ & $(2,11)$ & $\chi_{7}^{2}$ & \\
\hline 12 & \multirow{2}{*}{8505} & \multirow{2}{*}{$X^{3}-27 X-51$} & \multirow{2}{*}{$(7,15)$} & $\chi_{7}^{2}$ & \\
\hline 13 & & & & $\psi_{9}$ & \\
\hline
\end{tabular}

In the next section we will compute the relative class numbers of the six remaining fields numbered 2, 4, 8, 11, 12 and 13 in Table 2, thus proving Theorem 1(2) (see the results of our computations in Table 3 below).

4. Computation of relative class numbers. Let $N_{3} / M$ and $N_{4} / M$ be two other cubic subextensions of the abelian extension $K / M$. Then $N_{3}$ and $N_{4}$ are non-normal isomorphic CM-fields. Therefore, $h_{N_{4}}^{-}=h_{N_{3}}^{-}$.

Now, using abelian $L$-series associated with the abelian extension $K / L$, one can easily prove that

$$
\zeta_{K} / \zeta_{K^{+}}=\left(\prod_{i=1}^{4} \zeta_{N_{i}} / \zeta_{N_{i}^{+}}\right) /\left(\zeta_{M} / \zeta_{L}\right)^{3}
$$

Since $[K: M]$ and $\left[N_{i}: M\right]$ for $1 \leq i \leq 4$ are odd, we have $Q_{N_{i}}=Q_{K}=Q_{M}$, $h_{M}^{-}$divides $h_{K}^{-}$and each $h_{N_{i}}^{-}$for $1 \leq i \leq 4$. Since $\omega_{K}=\omega_{N_{2}}$ and $\omega_{N_{i}}=\omega_{M}$ for $1 \leq i \leq 3,(*)$ yields (see [Lou1])

$$
h_{K}^{-} / h_{M}^{-}=\prod_{i=1}^{4} h_{N_{i}}^{-} / h_{M}^{-} .
$$

Finally, let $\chi$ denote any one of the two sextic characters associated with the sextic cyclic extension $N_{3} / L$. Then

$$
\left(\text { ***) } \quad h_{N_{4}}^{-} / h_{M}^{-}=h_{N_{3}}^{-} / h_{M}^{-}=\frac{d_{L} N_{L / \mathbb{Q}}\left(\mathfrak{F}_{\chi}\right)}{(2 \pi)^{4}}|L(1, \chi)|^{2}=\left|\frac{1}{4} L(0, \chi)\right|^{2} .\right.
$$


Notice that if $h_{K}^{-}=1$, then $h_{M}^{-}=h_{N_{1}}^{-}=h_{N_{2}}^{-}=1$. Hence, according to $(* *)$ and $(* * *)$ we deduce that $h_{K}^{-}=\left(h_{N_{3}}^{-}\right)^{2}$ is a perfect square. Now we can take $\chi=\psi_{1} \psi_{2} \chi_{M}$ where $\psi_{1}, \psi_{2}$ and $\chi_{M}$ are the primitive abelian Hecke characters associated with the abelian extensions $N_{1}^{+} / L, N_{2}^{+} / L$, and $M / L$, respectively. It remains to evaluate $L(1, \chi)=L\left(1, \psi_{1} \psi_{2} \chi_{M}\right)$.

Theorem 2 ([Lou1, Theorem 4]). Let $\gamma=0.577215 \ldots$ denote Euler's constant and let $B$ be positive. Set

$$
K_{1}(B)=1+4 \sum_{n \geq 0}\left(\gamma+\log B-\frac{1}{2 n+2}-\sum_{k=1}^{n} \frac{1}{k}\right) \frac{B^{2 n+2}}{(2 n+2)(n !)^{2}}
$$

and

$$
K_{2}(B)=\pi B+4 \sum_{n \geq 0}\left(\gamma+\log B-\frac{1}{2 n+1}-\sum_{k=1}^{n} \frac{1}{k}\right) \frac{B^{2 n+2}}{(2 n+1)(n !)^{2}} .
$$

Then $B>0$ implies $0 \leq K_{2}(B) \leq K_{1}(B) \leq 2 e^{-B}$.

Let $L(s, \chi)=\sum_{n>1} a_{n}(\chi) / n^{s}$ be a Hecke $L$-series associated with a primitive Hecke character on a ray class group of a real quadratic number field L. Assume that $\chi$ is ramified at the infinite places $\infty_{1}$ and $\infty_{2}$ of L. Let $\infty_{1} \infty_{2} \mathfrak{F}_{\chi}$ be the conductor of $\chi$, set $f_{\chi}=N_{L / \mathbb{Q}}\left(\mathfrak{F}_{\chi}\right)$ and $A_{\chi}=\sqrt{d_{L} f_{\chi} / \pi^{2}}$. Let $W_{\chi}$ be the Artin root number associated with this L-series, i.e., let $W_{\chi}$ be the complex number of absolute value 1 such that

$$
F(1-s, \chi)=W_{\chi} F(s, \bar{\chi})
$$

where $F(s, \chi)=A_{\chi}^{s} \Gamma^{2}((s+1) / 2) L(s, \chi)$.

Then we have the following absolutely convergent series expansion:

$$
L(1, \chi)=\sum_{n \geq 1} \frac{a_{n}(\chi)}{n} K_{1}\left(n / A_{\chi}\right)+W_{\chi} \sum_{n \geq 1} \frac{\overline{a_{n}(\chi)}}{n} K_{2}\left(n / A_{\chi}\right) .
$$

If we let $S_{M, \chi}$ denote the value obtained by disregarding the indices $n>M$ in the above series, then

$$
\left|L(1, \chi)-S_{M, \chi}\right| \leq 4(\log (M e)+2)^{2} e^{-M / A_{\chi}} .
$$

It remains to compute $a_{n}(\chi)$ and $W_{\chi}$.

4.1. Computation of $\chi(I)=\psi_{1} \psi_{2} \chi_{M}(I)$ for non-zero integral ideals $I$. For any Hecke character $\chi$ there exists a character $\hat{\chi}$ on $\left(O_{L} / \mathfrak{F}_{\chi}\right)^{*}$ such that for any $\alpha \neq 0$ in $O_{L}, \chi((\alpha))=\nu(\alpha) \widehat{\chi}(\alpha)$, where $\nu(\alpha)$ is the sign of $N_{L / \mathbb{Q}}(\alpha)$. This character $\hat{\chi}$ is called the modular character associated with $\chi$. We describe explicitly the modular character $\widehat{\psi}_{2}$ for the six fields of degree 36 given in Table 2. (For $\psi_{1}$ and $\chi_{M}$ see [Lou1, Section 4.2] and [Lef, Section 2].) 
(1) $K=N_{1} N_{2}: N_{1}=E(\sqrt{-1}, \sqrt{-3})$ with $d_{E}=2^{2} \cdot 3^{3} \cdot 5, N_{2}=$ $k(\sqrt{-1}, \sqrt{-3})$ with $f_{k}=3^{2}$, and $L=\mathbb{Q}(\sqrt{3})$. We have $\mathfrak{F}_{N_{1}^{+} / L}=(15)$, $\mathfrak{F}_{M / L}=\infty_{1} \infty_{2}$, and $\mathfrak{F}_{N_{2}^{+} / L}=(3 \sqrt{3})$. Then $\left(O_{L} /(3 \sqrt{3})\right)^{*}=\left\{\varepsilon_{L}^{i} 4^{j} \mid 0 \leq\right.$ $i \leq 5,0 \leq j \leq 2\} \simeq \mathbb{Z} / 6 \mathbb{Z} \times \mathbb{Z} / 3 \mathbb{Z}, \widehat{\psi}_{2}\left(\varepsilon_{L}\right)=1$, and $\widehat{\psi}_{2}(4)=\zeta_{3}$, where $\varepsilon_{L}=2+\sqrt{3}$.

(2) $K=N_{1} N_{2}: N_{1}=E(\sqrt{-3}, \sqrt{-7})$ with $d_{E}=2^{2} \cdot 3^{3} \cdot 7, N_{2}=$ $k(\sqrt{-3}, \sqrt{-7})$ with $f_{k}=3^{2}$, and $L=\mathbb{Q}(\sqrt{21})$. We have $\mathfrak{F}_{N_{1}^{+} / L}=(6)$, $\mathfrak{F}_{M / L}=\infty_{1} \infty_{2}$, and $\mathfrak{F}_{N_{2}^{+} / L}=\mathfrak{p}^{3}$ with $(3)=\mathfrak{p}^{2}$ and $\mathfrak{p}=\left(\frac{3+\sqrt{21}}{2}\right)$. Then $\left(O_{L} / \mathfrak{p}^{3}\right)^{*}=\left\{\left(-\varepsilon_{L}\right)^{i} 4^{j} \mid 0 \leq i \leq 5,0 \leq j \leq 2\right\} \simeq \mathbb{Z} / 6 \mathbb{Z} \times \mathbb{Z} / 3 \mathbb{Z}, \widehat{\psi}_{2}\left(-\varepsilon_{L}\right)=1$, and $\widehat{\psi}_{2}(4)=\zeta_{3}$, where $\varepsilon_{L}=(5+\sqrt{21}) / 2$.

(3) $K=N_{1} N_{2}: N_{1}=E(\sqrt{-3}, \sqrt{-15})$ with $d_{E}=2^{2} \cdot 3^{4} \cdot 5, N_{2}=$ $k(\sqrt{-3}, \sqrt{-15})$ with $f_{k}=3^{2}$, and $L=\mathbb{Q}(\sqrt{5})$. We have $\mathfrak{F}_{N_{1}^{+} / L}=(18)$, $\mathfrak{F}_{M / L}=\infty_{1} \infty_{2}(3)$, and $\mathfrak{F}_{N_{2}^{+} / L}=(3)^{2}$. Then $\left(O_{L} /(3)^{2}\right)^{*}=\left\{\varepsilon_{L}^{i} 4^{j} \mid 0 \leq\right.$ $i \leq 23,0 \leq j \leq 2\} \simeq \mathbb{Z} / 24 \mathbb{Z} \times \mathbb{Z} / 3 \mathbb{Z}, \widehat{\psi}_{2}\left(\varepsilon_{L}\right)=1, \widehat{\psi}_{2}(4)=\zeta_{3}$, where $\varepsilon_{L}=(1+\sqrt{5}) / 2$.

(4) $K=N_{1} N_{2}: N_{1}=E(\sqrt{-2}, \sqrt{-11})$ with $d_{E}=2^{3} \cdot 7^{2} \cdot 11, N_{2}=$ $k(\sqrt{-2}, \sqrt{-11})$ with $f_{k}=7$, and $L=\mathbb{Q}(\sqrt{22})$. We have $\mathfrak{F}_{N_{1}^{+} / L}=(7)$, $\mathfrak{F}_{M / L}=\infty_{1} \infty_{2}, \mathfrak{F}_{N_{2}^{+} / L}=(7)$, and $(7)=\mathfrak{p}_{7} \mathfrak{p}_{7}^{\prime}$ with $\mathfrak{p}_{7}=7 \mathbb{Z}+(1+\sqrt{22}) \mathbb{Z}$ and $\mathfrak{p}_{7}^{\prime}=7 \mathbb{Z}+(1-\sqrt{22}) \mathbb{Z}$. If $\alpha=x_{\alpha}+y_{\alpha} \sqrt{22}$, then $\alpha \equiv x_{\alpha}-y_{\alpha}\left(\bmod \mathfrak{p}_{7}\right)$ and $\alpha \equiv x_{\alpha}-y_{\alpha}\left(\bmod \mathfrak{p}_{7}^{\prime}\right)$. Let $\phi$ be a character of order 3 on $\left(O_{L} / \mathfrak{p}_{7}\right)^{*}$. Then $\widehat{\psi}_{2}(\alpha)=\phi\left(\alpha \alpha^{\prime}\right)$, where $\alpha^{\prime}=x_{\alpha}-y_{\alpha} \sqrt{22}$.

(5) $K=N_{1} N_{2}: N_{1}=E(\sqrt{-7}, \sqrt{-15})$ with $d_{E}=3^{5} \cdot 5 \cdot 7, N_{2}=$ $k(\sqrt{-7}, \sqrt{-15})$ with $f_{k}=7$, and $L=\mathbb{Q}(\sqrt{105})$. We have $\mathfrak{F}_{N_{1}^{+} / L}=(9)$, $\mathfrak{F}_{M / L}=\infty_{1} \infty_{2}, \mathfrak{F}_{N_{2}^{+} / L}=\mathfrak{p}_{7}$, and $(7)=\mathfrak{p}_{7}^{2}$, where $\mathfrak{p}_{7}=7 \mathbb{Z}+\frac{7+\sqrt{105}}{2} \mathbb{Z}$. For any $\alpha=\left(x_{\alpha}+y_{\alpha} \sqrt{105}\right) / 2$ coprime to $\mathfrak{p}_{7}$ we have $\alpha \equiv\left(x_{\alpha}-7 y_{\alpha}\right) / 2$ $\left(\bmod \mathfrak{p}_{7}\right)$. Let $n_{\alpha}$ be an integer such that $n_{\alpha} \equiv\left(x_{\alpha}-7 y_{\alpha}\right) / 2(\bmod 7)$. Then $\widehat{\psi}_{2}(\alpha)=\zeta_{3}^{k}$, where $k=\min \left\{j \geq 0: n_{\alpha} \equiv 3^{j}(\bmod 7)\right\}$.

(6) $K=N_{1} N_{2}: N_{1}=E(\sqrt{-7}, \sqrt{-15})$ with $d_{E}=3^{5} \cdot 5 \cdot 7, N_{2}=$ $k(\sqrt{-3}, \sqrt{-15})$ with $f_{k}=3^{2}$, and $L=\mathbb{Q}(\sqrt{105})$. Then $\mathfrak{F}_{N_{1}^{+} / L}$ and $\mathfrak{F}_{M / L}$ are the same as (5). We have $\mathfrak{F}_{N_{2}^{+} / L}=\mathfrak{p}_{3}^{3}$ with $(3)=\mathfrak{p}_{3}^{2},\left(O_{L} / \mathfrak{p}_{3}^{3}\right)^{*}=\left\{\varepsilon_{L}^{i} 4^{j} \mid\right.$ $0 \leq i \leq 5,0 \leq j \leq 2\} \simeq \mathbb{Z} / 6 \mathbb{Z} \times \mathbb{Z} / 3 \mathbb{Z}, \widehat{\psi}_{2}\left(\varepsilon_{L}\right)=1$, and $\widehat{\psi}_{2}(4)=\zeta_{3}$, where $\varepsilon_{L}=41+4 \sqrt{105}$.

4.2. Computation of $a_{n}(\chi)$. Set $\chi=\psi_{1} \psi_{2} \chi_{M}$. Since $n \mapsto a_{n}(\chi)=$ $\sum_{N_{L / \mathbb{Q}}(I)=n} \chi(I)$ is multiplicative we only have to explain how to compute $a_{l^{k}}(\chi)$ on powers of primes ([Lou1]). Let $L_{0}$ and $L_{1}$ be two imaginary quadratic subfields of $M$ and let $\chi_{L_{0}}, \chi_{L_{1}}, \chi_{L}$ be quadratic Dirichlet characters associated with $L_{0}, L_{1}$, and $L$, respectively. 
Set $f=\operatorname{lcm}\left(N_{L / \mathbb{Q}}\left(\mathfrak{F}_{N_{1}^{+} / L}\right), N_{L / \mathbb{Q}}\left(\mathfrak{f}_{M / L}\right), N_{L / \mathbb{Q}}\left(\mathfrak{F}_{N_{2}^{+} / L}\right)\right)$ and

$$
\eta_{l}= \begin{cases}0 & \text { if } \chi_{L_{0}}(l)=\chi_{L_{1}}(l)=0 \\ -1 & \text { if } \chi_{L}(l) \neq-1 \text { and if either } \chi_{L_{0}}(l)=-1 \text { or } \chi_{L_{1}}(l)=-1 \\ 1 & \text { otherwise, }\end{cases}
$$

where $\mathfrak{f}_{M / L}$ denotes the finite part of the conductor $\mathfrak{F}_{M / L}$. If $l \mid f$, then $a_{l^{k}}(\chi)=0$. Assume that $(l, f)=1$.

(a) If $\chi_{L}(l)=-1$, then $\psi_{1}(l)=\chi_{M}(l)=1$ and

$$
a_{l^{k}}(\chi)= \begin{cases}0 & \text { if } k \text { is odd } \\ \psi_{2}(l)^{k / 2} & \text { if } k \text { is even. }\end{cases}
$$

(b) If $\chi_{L}(l)=0$ and $(l)=\mathfrak{L}^{2}$, then $\psi_{1}(\mathfrak{L})=1$ and $a_{l^{k}}(\chi)=\eta_{l}^{k} \psi_{2}(\mathfrak{L})^{k}$.

(c) If $\chi_{L}(l)=1$ and $(l)=\mathfrak{L} \mathfrak{L}^{\prime}$, then $1=\psi_{1}((l))=\psi_{1}(\mathfrak{L}) \psi_{1}\left(\mathfrak{L}^{\prime}\right)$ and $\psi_{2}((l))=\psi_{2}(\mathfrak{L}) \psi_{2}\left(\mathfrak{L}^{\prime}\right)$. We have

$$
\begin{aligned}
a_{l^{k}}(\chi) & =\sum_{i=0}^{k} \chi(\mathfrak{L})^{i} \chi\left(\mathfrak{L}^{\prime}\right)^{k-i} \\
& =\eta_{l}^{k} \sum_{i=0}^{k} \psi_{1}(\mathfrak{L})^{i} \psi_{2}(\mathfrak{L})^{i} \psi_{1}\left(\mathfrak{L}^{\prime}\right)^{k-i} \psi_{2}\left(\mathfrak{L}^{\prime}\right)^{k-i} \\
& =\eta_{l}^{k} \sum_{i=0}^{k} \psi_{1}(\mathfrak{L})^{2 i-k} \psi_{2}((l))^{k-i} \psi_{2}(\mathfrak{L})^{2 i-k} .
\end{aligned}
$$

Then $\mathfrak{L}^{h_{L}}=(\alpha)$ for some integer. We have $\psi_{1}(\mathfrak{L})=\widehat{\psi}_{1}(\alpha)^{h^{\prime}}$ and $\psi_{2}(\mathfrak{L})=$ $\widehat{\psi}_{2}(\alpha)^{h^{\prime}}$ if $\left(h_{L}, 3\right)=1$ and $h_{L} h^{\prime} \equiv 1(\bmod 3)$.

4.3. Numerical computation of approximations of the Artin root numbers. We recall Louboutin's ideas for computing approximations of the Artin root numbers (see [Lou1, Section 5]). Set

$$
G(x, \chi)=\frac{1}{2 i \pi} \int_{\alpha-i \infty}^{\alpha+i \infty} F(s, \chi) x^{-s} d s \quad \text { with } x>0 \text { and } \alpha>1
$$

and

$$
H(B)=-4 \sum_{n \geq 0} \frac{B^{2 n+1}}{(n !)^{2}}\left(\gamma+\log B-\sum_{k=1}^{n} \frac{1}{k}\right) \quad \text { for } B>0
$$

(where $\gamma=0.577 \ldots$ denotes Euler's constant). Then

$$
G(x, \chi)=\sum_{n \geq 1} a_{n}(\chi) H\left(n x / A_{\chi}\right)
$$

(where $A_{\chi}$ is as in Theorem 2), and we can therefore easily compute good approximations of $G(1, \chi)$. If we can deduce from these approximations that 
$G(1, \chi) \neq 0$, then since

$$
G(x, \chi)=\frac{W_{\chi}}{x} G(1 / x, \bar{\chi}),
$$

we have $W_{\chi}=G(1, \chi) / \overline{G(1, \chi)}$ and obtain good approximations of $W_{\chi}$ which can be used, together with Theorem 2, to compute good approximations of the integer $h_{K}^{-}$, hence to compute its exact value. According to our numerical computations for the six fields given in Table 2 we have $G(1, \chi) \neq 0$ and we obtain Table 3 .

Table 3

\begin{tabular}{|c|c|c|c|c|c|c|c|}
\hline$d_{E}$ & $\left(m_{0}, m_{1}\right)$ & $f_{k}$ & $W_{\chi}$ & $W_{\chi}^{6}$ & $L(1, \chi)$ & $L(0, \chi) / 4$ & $h_{K}^{-}$ \\
\hline 2700 & $(1,3)$ & 9 & $\begin{array}{l}-0.642787 \ldots \\
-0.766044 \ldots i\end{array}$ & $\frac{1-\sqrt{-3}}{2}$ & $\begin{array}{l}1.013996 \ldots \\
-0.564536 \ldots i\end{array}$ & $\frac{-1-3 \sqrt{-3}}{2}$ & $7^{2}$ \\
\hline 756 & $(3,7)$ & 9 & $\begin{array}{l}-0.642787 \ldots \\
-0.766044 \ldots i\end{array}$ & $\frac{1-\sqrt{-3}}{2}$ & $\begin{array}{l}0.816374 \ldots \\
-0.143948 \ldots i\end{array}$ & $\frac{-1-\sqrt{-3}}{2}$ & 1 \\
\hline \multirow{2}{*}{1620} & \multirow{2}{*}{$(3,15)$} & 9 & $\begin{array}{l}-0.766044 \ldots \\
+0.642787 \ldots i\end{array}$ & $\frac{-1+\sqrt{-3}}{2}$ & $\begin{array}{l}0.921696 \ldots \\
+0.335470 \ldots i\end{array}$ & $\frac{-1+\sqrt{-3}}{2}$ & 1 \\
\hline \multirow{2}{*}{4312} & \multirow{2}{*}{$(2,11)$} & 7 & $\begin{array}{l}0.386513 \ldots \\
+0.922283 \ldots i\end{array}$ & $\frac{71+39 \sqrt{-3}}{2 \cdot 7^{2}}$ & $\begin{array}{l}0.232372 \ldots \\
+0.554478 \ldots i\end{array}$ & 1 & 1 \\
\hline \multirow{2}{*}{8505} & \multirow{2}{*}{$(7,15)$} & 7 & $\begin{array}{l}-0.895953 \ldots \\
-0.444148 \ldots i\end{array}$ & $\frac{-13+3 \sqrt{-3}}{14}$ & $\begin{array}{l}1.325166 \ldots \\
+0.969708 \ldots i\end{array}$ & $-10+\sqrt{-3}$ & $103^{2}$ \\
\cline { 2 - 8 } & & 9 & $\begin{array}{l}0.766044 \ldots \\
+0.642787 \ldots i\end{array}$ & $\frac{-1-\sqrt{-3}}{2}$ & $\begin{array}{l}1.788302 \ldots \\
+0.532666 \ldots i\end{array}$ & $4+\sqrt{-3}$ & $19^{2}$ \\
\hline
\end{tabular}

REMARK. Since $h_{M}^{-}=h_{N_{1}}^{-}=h_{N_{2}}^{-}=1$ for those six fields, we have $h_{K}^{-}=\left(h_{N_{3}}^{-} / h_{M}^{-}\right)^{2}=\left|\frac{1}{4} L(0, \chi)\right|^{4}$. According to Siegel-Klingen's Theorem ([Hid, Cor. 1 in $\S 2.5]) L(0, \chi) \in \mathbb{Q}(\sqrt{-3})$. Using [Lou2] we can determine explicitly $L(0, \chi)$. Moreover, we can prove that $W_{\chi}^{6} \in \mathbb{Q}(\sqrt{-3})$.

Acknowledgements. The authors are greatly indebted to S. Louboutin for his many helpful suggestions during the preparation of the paper. In particular, the proof of Proposition 1 and the factorization of $h_{K}^{-}$in Section 4 are much improved owing to his suggestions. This research was supported by Grant BK21.

\section{References}

[CK] K.-Y. Chang and S.-H. Kwon, Class numbers of imaginary abelian number fields, Proc. Amer. Math. Soc. 128 (2000), 2517-2528.

[H] J. Hoffstein, Some analytic bounds for zeta functions and class numbers, Invent. Math. 55 (1979), 37-47. 
[Hid] H. Hida, Elementary Theory of L-functions and Eisenstein Series, London Math. Soc. Stud. Texts 26, Cambridge Univ. Press, 1993.

[K] M. Daberkow, C. Fieker, J. Klüners, M. Pohst, K. Roegner and K. Wildanger, KANT V $V_{4}$, J. Symbolic Comput. 24 (1997), 267-283.

[Lef] Y. Lefeuvre, Corps diédraux à multiplication complexe principaux, Ann. Inst. Fourier (Grenoble) 50 (2000), 67-103.

[LLO] F. Lemmermeyer, S. Louboutin and R. Okazaki, The class number one problem for some non-abelian normal CM-fields of degree 24, J. Théor. Nombres Bordeaux 11 (1999), 387-406.

[Lou1] S. Louboutin, Computation of relative class numbers of CM-fields by using Hecke L-functions, Math. Comp. 69 (2000), 371-393.

[Lou2] - Computation of $L(0, \chi)$ and of relative class numbers of CM-fields, Nagoya Math. J. 161 (2001), 171-191.

[LOO] S. Louboutin, R. Okazaki and M. Olivier, The class number one problem for some non-abelian normal CM-fields, Trans. Amer. Math. Soc. 349 (1997), 3657-3678.

[O] A. M. Odlyzko, Some analytic estimates of class numbers and discriminants, Invent. Math. 29 (1975), 37-47.

[P] Y.-H. Park, The class number one problem for the non-abelian normal CM-fields of degree 24 and 40, this issue, 63-80.

[W] L. C. Washington, Introduction to Cyclotomic Fields, 2nd ed., Grad. Texts in Math. 83, Springer, 1997.

[Y] K. Yamamura, The determination of the imaginary abelian number fields with class number one, Math. Comp. 62 (1994), 899-921.

Information Security Basic Research Team

ETRI

161 Kajong-dong, Yusong-Gu

305-350 Taejon, South Korea

E-mail: jang1090@etri.re.kr
Department of Mathematics Education Korea University 136-701 Seoul, South Korea E-mail: shkwon@semi.korea.ac.kr

Received on 8.9.2000

and in revised form on 10.1.2001 